The Impact of Large-Scale Surveys on Pulsating Star Research

ASP Conference Series, Vol. 203, 2000

L. Szabados \& D. W. Kurtz, eds.

\title{
Cepheid Distance to the Virgo Cluster
}

\author{
Anwesh Mazumdar, D. Narasimha \\ Tata Institute of Fundamental Research, Mumbai 400005, India
}

\begin{abstract}
A reanalysis of the HST data on Cepheids in the Virgo cluster spiral M100 is presented, based on our study of the Galactic Cepheids. A distance modulus of $31.49 \mathrm{mag}$ is derived, which is higher than the HST Key Project value of 31.04 mag obtained from the same data earlier (Ferrarese et al. 1996). The increase in the distance is caused by nearly equal contributions from a different calibration of the slope and the zero point of the period-luminosity relation and a correction for the flux-limited incompleteness bias.
\end{abstract}

The calibration of the Cepheid period-luminosity relation (PLR) as a primary distance indicator is reviewed. The conventional method of determining the slope of the PLR is biased heavily by the numerous short period Cepheids in the calibrating local sample. But in distant galaxy observations, these are missed due to flux limitation, and the observed sample mostly consists of longer period Cepheids which are easily detected. It is therefore important to calibrate the PLR for the longer period range in order to apply it to distant galaxies. From the best fits to observed period-magnitude diagrams for Cepheids in the Galaxy, LMC and IC4182 we find that the longer period Cepheids obey a PLR with a slope which is steeper than the generally accepted value of -2.77 . The mean value of the slope derived from these galaxies is $-3.25 \pm 0.25$ for periods between 15 and $60 \mathrm{~d}$.

In view of the problems with the distance determination to LMC regarding metallicity and reddening effects, and the wide range of values obtained from different distance indicators, we chose to have additional local calibration of the zero point of the PLR based on Hipparcos observations. Summarizing all the results from local and LMC Cepheids, we adopted the zero point value of $M_{V}=-4.23$ mag for a 10 -d-period Cepheid.

At the first step of our analysis of the M100 Cepheids, we find the best fit PLR to the observed data, prior to any correction for extinction and incompleteness. This ensures that this analysis is subject to minimum number of assumptions regarding any kind of corrections. The best fits are obtained separately for long and short period Cepheids. We find that the longer period Cepheids $(1.47<\log P \leq 1.85)$ obey a Gaussian distribution with a slope of $-3.15 \pm 0.15$, in excellent agreement with the local calibration. The derived true distance modulus is $31.56 \pm 0.4 \mathrm{mag}$, which agrees well with our final value, and proves that our final distance estimate is not an artifact of our data analysis technique. This sub-sample $(\log P>1.47)$ can be used to obtain a fairly unbiased distance modulus of M100 directly without invoking other systematic corrections. On the other hand, Cepheids with $1.15<\log P \leq 1.47$ have a non-Gaussian and 

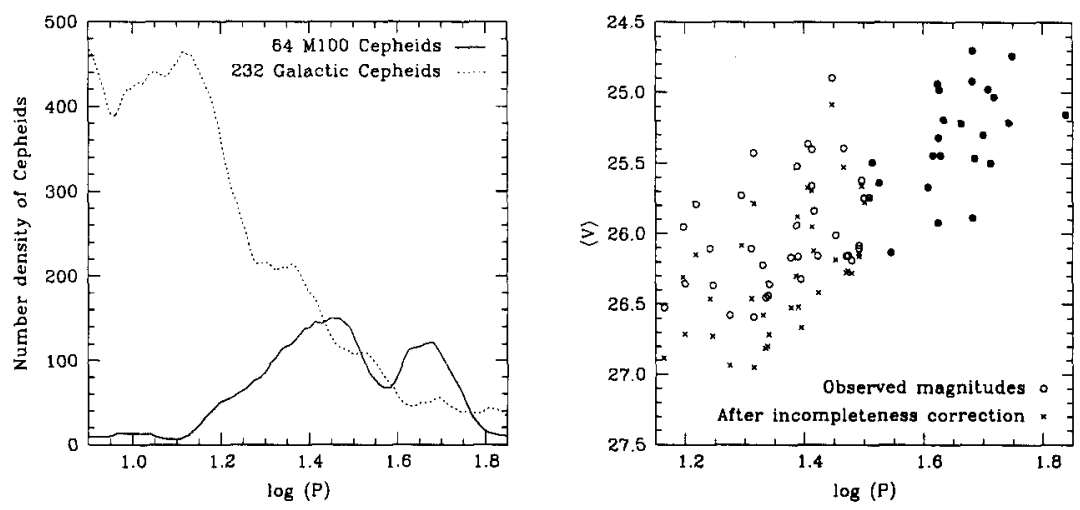

Figure 1. Left: The observed frequency distribution of Cepheids in our Galaxy and M100 shows that M100 has fewer number of Cepheids at short periods due to flux limitation. Right: The incompleteness correction adopted for the short period range in the M100 sample.

asymmetric distribution about the best-fit line, and the slope varies between a wide range of +0.2 to -2.2 , depending on the choice of the sample and the minimization technique. The derived distance modulus is also smaller than that for the long period Cepheids. We conclude that this sub-sample suffers from a magnitude-dependent incompleteness bias.

For the full sample with $1.15<\log P \leq 1.85$, the slope is $-2.2 \pm 0.2$, which is shallower than that of other galaxies for a similar period range, because the fainter short period Cepheids have been missed due to flux limitation, and consequently the average brightness at a given period is overestimated in the short period range. To utilize the full sample of Cepheids, we must correct for this incompleteness bias. This bias is clearly seen in the comparative study of number density of observed Cepheids with respect to $\log P$ of the Galaxy and M100. At periods shorter than 30 days, the number of observed M100 Cepheids goes down progressively in comparison to the Galactic Cepheids (Fig. 1). The correction for incompleteness is based on a simple mathematical analysis of the number density distribution of M100 Cepheids, utilizing the scatter in the PLR. The bias is offset by making the average magnitude fainter by the calculated amount at short periods (Fig. 1). No correction is needed for Cepheids with periods longer than $35 \mathrm{~d}$. But at shorter periods $(\sim 20 \mathrm{~d})$ a correction $\sim 0.36$ mag is necessary.

The extinction correction is based on period-color-amplitude relations derived for Galactic Cepheids. Comparing the final $\log P-\langle V\rangle_{0}$ magnitude relation with our adopted PLR, we estimate the distance modulus to M100 to be 31.49 mag. The major contributions to the error in the analysis are from the calibration of the PLR and the corrections for incompleteness and extinction. The final distance estimate to M100 from the present analysis is $19.9 \pm 1.7$ (random) \pm 2.4 (systematic) Mpc.

\section{Reference}

Ferrarese, L., Freedman, W. L., Hill, R. J., et al. 1996, ApJ, 464, 568 